\title{
OBLIQUE SHOCKS IN EXTRAGALACTIC JETS
}

\author{
D. FRAIX-BURNET \\ Observatoire Midi-Pyrénées \\ 14 Avenue Edouard Belin \\ $\$ 1400$ Toulouse \\ France
}

In the framework of the diffusive shock acceleration of relativistic electrons in extragalactic jets, we show that it is possible to derive the speed of the jet. For this purpose, we transform continuity relations through an oblique shock front into the reference frame of the observer. We apply these calculations to knot A of the M87 jet. Measuring the deviation of the fluid through the shock front from high-resolution radio maps and the deviation of the magnetic field from optical polarization maps (Fraix-Burnet et al., 1989), we derive speeds of about 0.01 c (Fraix-Burnet and Biermann, in preparation). The compression ratio is most probably 4 and the magnetic field is nearly parallel to the shock front both upstream and downstream.

In addition, it can be shown that the diffusive shock acceleration mechanism is still possible when the shock front speed projected along the jet direction is larger than c. The immediate application of these calculations is the production of apparent superluminal motions (Fraix-Burnet, 1989) that could explain VLBI jets.

The influence of shock obliquity in the diffusive shock acceleration mechanism is also studied. In particular, we derive the variations with obliquity of the spectral index and of the cut-off frequency. For the spectral index, we generalize to oblique shocks the analytical model developed by Pelletier and Roland $(1986,1988)$. With the observed range of radio spectral index (Bridle and Perley, 1984), we conclude that the Alfvénic Mach number is certainly not larger than 30, and that the pressure of the relativistic particles is roughly equal to that of the thermal plasma (Fraix-Burnet and Pelletier, in preparation). The cut-off frequency is obtained by equating the characteristic acceleration time and the synchrotron loss time. The obliquity turns out to have no influence under $60^{\circ}$ (Fraix-Burnet and Pelletier, in preparation). With a Kraichnan spectrum of the magnetic field turbulences, we find that the level of these turbulences must be very low to account for the very few observed cut-off frequencies in the optical.

Bridle, A.H., Perley, R.A.: 1984, Ann. Rev. Astron. Astrophys. 22, 319

Fraix-Burnet, D.: 1989, Astron. Astrophys. in press

Fraix-Burnet, D., Le Borgne, J.-F., Nieto, J.-L.: 1989, Astron. Astrophys. in press

Pelletier, G., Roland, J.: 1986, Astron. Astrophys. 163, 9

Pelletier, G., Roland, J.: 1988, Astron. Astrophys. 196, 71 